\title{
A Chinese Herbal Preparation, Xiao-Er-An-Shen Decoction, Exerts Neuron Protection by Modulation of Differentiation and Antioxidant Activity in Cultured PC12 Cells
}

\author{
Zhonggui Li $\left(\mathbb{D},{ }^{1}\right.$ Kelly Y. C. Lam, ${ }^{2}$ Xiaoyan Liu, ${ }^{1}$ Airong Qi, ${ }^{1}$ Ping Yao $\left(\mathbb{D},{ }^{2}\right.$ \\ Tina T. X. Dong $\mathbb{D},^{2}$ Tiegang Yi, ${ }^{1}$ Karl W. K. Tsim $\mathbb{D}^{2},{ }^{2}$ and Jianping Chen $\mathbb{D}{ }^{1}$ \\ ${ }^{1}$ Shenzhen Key Laboratory of Hospital Chinese Medicine Preparation, Shenzhen Traditional Chinese Medicine Hospital, \\ Guangzhou University of Chinese Medicine, Shenzhen, China \\ ${ }^{2}$ Division of Life Science and Center for Chinese Medicine, The Hong Kong University of Science and Technology, Kowloon, Hong Kong \\ Correspondence should be addressed to Jianping Chen; lycjp@126.com
}

Received 31 July 2017; Revised 7 March 2018; Accepted 4 April 2018; Published 3 May 2018

Academic Editor: Eman Al-Sayed

Copyright (C) 2018 Zhonggui Li et al. This is an open access article distributed under the Creative Commons Attribution License, which permits unrestricted use, distribution, and reproduction in any medium, provided the original work is properly cited.

\begin{abstract}
Xiao-Er-An-Shen Decoction (XEASD), a Chinese herbal formula, has been used in clinic for treating insomnia and mental excitement in children and adolescents. However, less of scientific data supports its effectiveness in clinic. Here, we aim to study the role of XEASD in regulating neuron differentiation and antioxidant activity. An HPLC-MS was used to chemically standardize herbal extract of XEASD. The standardized herbal extracts of XEASD $(0.3-3.0 \mathrm{mg} / \mathrm{mL})$ were applied onto cultured PC12 cells for 48 hours. The treatment with XEASD extract induced neurite outgrowth of PC12 cells in a dose-dependent manner, having the highest response by $\sim 50 \%$ of differentiated cells. Application of XEASD extract dose dependently stimulated expressions of NF68, NF160, and NF200 in cultured PC12 cells. Furthermore, XEASD activated the phosphorylation of cAMP responsive element binding protein on PC12 cells, the effect of which was blocked by H89, a protein kinase A inhibitor. Moreover, XEASD showed free radical scavenging activity and stimulated the transcriptional activity of ARE. These results supported the neurobeneficial effects of XEASD in the induction of neurite outgrowth and protection against oxidative stress and could be useful for neurological diseases, in which neurotrophin deficiency and oxidation insult are involved.
\end{abstract}

\section{Introduction}

Sleep disturbances are widely spread in children and adolescents, and the prevalence of sleep-related problems was estimated to be $\sim 43 \%$ [1]. Insomnia is characterized by a difficulty in falling asleep and/or staying asleep, which is the most common sleep disorder with high rate of serious conditions [2]. It was reported that people with insomnia have a twofold risk to develop depression compared to people with no difficulty in falling asleep [3]. Moreover, it has been estimated that $90 \%$ of patients with depression complain about their sleep quality [4]. Sleep disorders are linked with potential suicidal behaviors in depressed youth [5]. Thus, sleep problems like insomnia should be taken as major concern among parents and doctors. Hypnotics are primarily used in clinic to treat insomnia, yet the treatment of this is accompanied by many side effects. This is not an ideal therapeutic regimen for children and adolescents. Therefore, there is a need to develop an alternative medicine for the treatment of insomnia in children and adolescents.

Neurogenesis is the process by which neurons are generated, including cell proliferation, survival, maturation, and differentiation [6]. The findings suggested that disturbance of sleep can lead to a reduction of neurogenesis. And a decrease in neurogenesis was also considered as one of the critical factors contributing to the pathophysiology of depression [7]. Brain development in children and adolescence is foundation upon which the brain continues developing, the impairment of which can result in a fragile and unreliable foundation. Taking together, we propose that enhancement of neurogenesis could be one of the effective approaches in improving 
TABLE 1: The composition and proportion of herb in XEASD.

\begin{tabular}{|c|c|c|c|}
\hline Botanical name & Herbal name & Chinese name & Dosage \\
\hline Polygala tenuifolia Willd. & Polygalae Radix & Yuan-Zhi & $16 \mathrm{~g}$ \\
\hline Astragalus membranaceus (Fisch.) Bge. var. mongholicus (Bge.) Hsiao & Astragali Radix & Huang-Qi & $16 \mathrm{~g}$ \\
\hline Acorus tatarinowii Schott & Acori Tatarinowii Rhizoma & Shi-Chang-Pu & $25 \mathrm{~g}$ \\
\hline Citrus reticulate Blanco & Citri Reticulatae Pericarpium & Chen-Pi & $8 \mathrm{~g}$ \\
\hline Alpinia oxyphylla Miq. & Alpiniae Oxyphyllae Fructus & Yi-Zhi & $16 \mathrm{~g}$ \\
\hline Citrus aurantium L. & Aurantii Fructus & Zhi-Qiao & $16 \mathrm{~g}$ \\
\hline Pinellia ternata (Thunb.) Breit. & Pinelliae Rhizoma & Ban-Xia & $16 \mathrm{~g}$ \\
\hline Notopterygium incisum Ting ex H. T. Chang & Notopterygii Rhizoma et Radix & Qiang-Huo & $8 \mathrm{~g}$ \\
\hline Glycyrrhiza uralensis Fisch. & Glycyrrhizae Radix et Rhizoma & Gan-Cao & $6 \mathrm{~g}$ \\
\hline
\end{tabular}

sleep problems and further preventing the development of depression for youth.

Xiao-Er-An-Shen Decoction (XEASD), a Chinese herbal formula, has been clinically prescribed to treat insomnia and mental excitement in children and adolescents, which possesses the efficacies of nourishing the brain to tranquilize and dispelling phlegm to settle fright. However, less is known about the molecular mechanism of XEASD in supporting its brain benefit. In our current studies, we investigated whether XEASD possessed neurogenesis role in neuronal cells. The effect of XEASD on neuron differentiation, an important stage of neurogenesis, was firstly evaluated. We determined the neurite outgrowth and neurofilament expression in neuronal PC12 cells, a wide model for the study of neuronal differentiation. In addition, the antioxidative role of XEASD was revealed to prevent the impairment of neurogenesis under oxidative stress.

\section{Materials and Methods}

2.1. Chemical and Drugs. XEASD was kindly provided by Pharmaceutical Department of Shenzhen Traditional Chinese Medicine Hospital. XEASD mainly consists of night herbs and the mixed proportion of respective herb is illustrated in Table 1. Liquiritin (1), calycosin 7-O- $\beta$-glucoside (2), ammonium glycyrrhizinate (3), naringin (4), 3,6' disinapoylsucrose (5), hesperidin (6), neohesperidin (7), and astragaloside IV (8) were purchased from National Institutes for Food and Drug Control (Beijing, China). The purities of all these chemicals were over 98\%. HPLC grade acetonitrile was purchased from Merck (Darmstadt, Germany), and ultrapure water was prepared using a Milli-Q purification system (Molsheim, France). Other reagents were of analytical grade.

2.2. Chromatographic Conditions and Instrumentation. A validation HPLC method was applied onto a Shimadzu (Kyoto, Japan) LC-20AT system. The herbal extract was separated on Agilent ZORBAX SB-C18 $(250 \mathrm{~mm} \times 4.6 \mathrm{~mm}, 5 \mu \mathrm{m})$ column. The mobile phase was composed of acetonitrile (A) and $10 \mathrm{mmol} / \mathrm{L}$ ammonium acetate (B) using the following gradient program: $0-0.01 \mathrm{~min}, 15 \% \mathrm{~A}$; $0.01-5 \mathrm{~min}, 15 \%-19 \%$ A; 5-22 $\min , 19 \%-35 \%$ A; $22-35 \mathrm{~min}, 35 \%-90 \%$ A; the flow rate was $0.8 \mathrm{ml} / \mathrm{min}$; the injection volume was $5 \mu \mathrm{l}$. A Shimadzu mass spectrum (LC-2020) equipped with an ESI ion source was performed in both positive and negative modes, and the selected ion monitoring was employed. The drying gas temperature was $350^{\circ} \mathrm{C}$; drying gas flow was $1.5 \mathrm{~L} / \mathrm{min}$; nebulizer pressure was 35 psi; capillary voltage was $3500 \mathrm{~V}$. Shimadzu Mass Workstation software was used for data acquisition and processing.

2.3. Cell Culture. Pheochromocytoma PC12 cells, a cell line derived from rat adrenal medulla, were purchased from American Type Culture Collection (ATCC, Manassas, VA). The cells were cultured in Dulbecco's modified Eagles medium (DMEM) supplemented with $6 \%$ fetal bovine serum (FBS), 6\% horse serum (HS), $100 \mathrm{U} / \mathrm{mL}$ penicillin, and $100 \mu \mathrm{g} / \mathrm{mL}$ streptomycin at $37^{\circ} \mathrm{C}$ in a water-saturated $7.5 \%$ $\mathrm{CO}_{2}$ incubator. Cultured medium was changed with fresh one every other day. Reagents for cell cultures were obtained from Invitrogen Technologies (Carlsbad, CA).

2.4. Neurite Outgrowth Assay. The assessment of neurite outgrowth in PC12 cells was performed according to previous report [8]. In brief, Cultured PC12 cells were treated with drugs for 48 hours and changed with medium containing XEASD every 24 hours. Nerve growth factor (NGF; Alomone Labs; Jerusalem, Israel) at concentration of $50 \mathrm{ng} / \mathrm{mL}$ was employed as a positive control. Cells were fixed with icecold $4 \%$ paraformaldehyde immediately after collection. A light microscope (Zeiss Group, Jena, Germany) equipped with a phase-contrast condenser, 10x objective lens, a digital camera, and SPOT imaging software was employed to capture and analyze the neurite presence and neurite length, and approximately 100 cells were counted from more than 10 randomly chosen visual fields per each culture. The cells were defined as differentiated if one or more neurites were longer than the diameter of cell body and also classified according to their neurite length in $<15 \mu \mathrm{m}, 15-30 \mu \mathrm{m}$, and $>30 \mu \mathrm{m}$.

\subsection{Sodium Dodecyl Sulfate Polyacrylamide Gel Electrophore-} sis (SDS-PAGE). To investigate neurofilament expression, PC1 2 cells $\left(8 \times 10^{4}\right.$ cells $\left./ \mathrm{mL}\right)$ were seeded onto 12 -well plates in normal serum medium for 24 hours, and then medium was transferred to low serum (1\% FBS, $1 \%$ HS, $100 \mathrm{U} / \mathrm{mL}$ penicillin, and $100 \mu \mathrm{g} / \mathrm{mL}$ streptomycin) as indicated for 3 hours prior to exposure XEASD. After 48 hours of treatment, the cultures were collected in high salt lysis buffer $(1 \mathrm{M}$ 
$\mathrm{NaCl}, 10 \mathrm{mM}$ HEPES, pH 7.5, $1 \mathrm{mM}$ EDTA, 1 mM EGTA, and $0.5 \%$ Triton X-100), followed by centrifugation at $16,100 \times \mathrm{g}$ for 10 mins at $4^{\circ} \mathrm{C}$. Total protein content was determined by Bradford's method with a kit from Bio-Rad Laboratories (Hercules, CA). Samples of equal amount of total protein were added with $2 \mathrm{x}$ direct lysis buffer $(0.125 \mathrm{M} \mathrm{HCl}, \mathrm{pH} 6.8$, $4 \%$ SDS, 20\% glycerol, 2\% 2-mercaptoethanol, and 0.02\% bromophenol blue) and boiled for 10 mins before being separated on $8 \%$ gel electrophoresis.

2.6. Protein Phosphorylation on PC12 Cells. PC12 cells were seeded onto 12-well plate. After the degree of confluence was over 90 percentage, the culture medium was changed to DMEM medium without serum over 5 hours. The cells were treated with XEASD or NGF at different time points in the absence or presence of $\mathrm{H} 89(0 \mathrm{~min}, 5 \mathrm{~min}, 10 \mathrm{~min}$, and $30 \mathrm{~min}$ ). Then, the cells were harvested, digested with $200 \mu \mathrm{L}$ of $2 \mathrm{x}$ direct lysis buffer, and boiled for $10 \mathrm{~min}$ before undergoing $8 \%$ gel electrophoresis.

2.7. Western Blot Analysis. Following the electrophoresis, proteins running on an $8 \%$ SDS-PAGEs were transferred to the nitrocellulose membrane, using a Mini Trans-Blot ${ }^{\circledR}$ cell at $40 \mathrm{~V}$ and $0.1 \mathrm{~A}$ for 16 hours in $1 \mathrm{x}$ transfer buffer with $24 \mathrm{mM}$ Tris, $192 \mathrm{mM}$ glycine, $15 \%$ ethanol, and $0.1 \%$ SDS. Transfer and equal loading of the samples were confirmed using Ponceau-S staining. After being blocked with 5\% fat-free milk in Tris-buffer saline/0.1\% Tween 20 (TBS-T), the membranes were incubated in the primary antibody diluted in $2.5 \%$ fat-free milk in TBS-T at $4^{\circ} \mathrm{C}$ overnight. The primary antibodies used were antineurofilament 200 (NF200; $1: 1,000$, Sigma), anti-NF160 (1:2,500, Sigma), antiNF68 (1:2,500, Sigma), and anti- GAPDH (1:10,000; Abcam Ltd., Cambridge, UK). Then, the membrane was rinsed with TBS-T and incubated for 2 hours at the room temperature in horseradish peroxidase- (HRP-) conjugated anti-mouse secondary antibody (Invitrogen) diluted in the $2.5 \%$ fatfree milk in TBS-T. The immune complexes were visualized using the enhanced chemiluminescence (ECL) method (GE Healthcare, Piscateway, NJ). The densitometric analysis of the bands in various samples was performed on an image analyzer.

2.8. DNA Construction and Transfection. The vector, pGL4.37 [luc2P/ARE/Hygro], contains four copies of an antioxidant response element (ARE, $5^{\prime}$-TAGCTTGGAA ATGACATTGC TAATGGTGAC AAAGCAACTT T- $3^{\prime}$ ) having a downstream luciferase reporter gene luc2P (Photinus pyralis) (Promega Corporation, Madison, WI). Cultured PC12 cells were transiently transfected with pARE-Luc using Lipofectamine 3000 (Thermo Fisher Scientific Inc, Waltham, MA), according to the manufacturer's guidelines. The cells were ready for experiments after 24 hours of transfection.

2.9. Luciferase and Protein Assays. After drug treatment, luciferase assay was analyzed by a commercial kit (Tropix Inc., Bedford, MA). Briefly, cultures were digested by a buffer containing $100 \mathrm{mM}$ potassium phosphate buffer $(\mathrm{pH}=7.8)$, $0.2 \%$ Triton X-100, and $1 \mathrm{mM}$ dithiothreitol. The luminescent reaction was measured in a Glomax ${ }^{\mathrm{TM}} 96$ Microplate Luminometer, and the activity was expressed as absorbance (up to $560 \mathrm{~nm}$ ) per mg of protein. Protein concentrations were determined routinely by Bradford's method with a kit from Bio-Rad Laboratories (Hercules, CA).

2.10. DPPH Radical Scavenging Assay. DPPH solution $(100 \mu \mathrm{g} / \mathrm{ml}, \quad 2,2$-diphenyl-1-picrylhydrazyl, Sigma) was prepared with methanol (HPLC grade). Fifty $\mu \mathrm{L}$ of extracts with different concentration together with $150 \mu \mathrm{L}$ of DPPH solution was added to each well of 96-well microplate. After incubating for $30 \mathrm{mins}$ at room temperature avoiding light, the absorbance was recorded at $495 \mathrm{~nm}$ for each sample tested against methanol blank using a spectrophotometer. The DPPH radical scavenging effect was expressed as \% scavenging relative to control (without samples).

2.11. Statistical Analysis. Statistical tests were analyzed using $T$-Test (version 13.0, SPSS, IBM Corporation, Armonk, NY). Statistically significant changes were classified as significant $(*)$ where $p<0.05$, more significant $(* *)$ where $p<0.01$, and highly significant $(* * *)$ where $p<0.001$.

\section{Results}

3.1. Chemical Standardization of XEASD. An HPLC-MS method was developed to reveal the chemical profile and quantify the main ingredients of XEASD. Eight chemical markers were identified in XEASD extract (Figure 1(a)). A typical LC-MS profile was developed for XEASD extract (Figure 1(b)), which was employed as a parameter for the identification of XEASD. Besides, eight chemical components were quantified in the XEASD extract defining the minimum requirement for $1 \mathrm{~g}$ of dried powder of standardized XEASD, that is, liquiritin $(0.13 \mathrm{mg} / \mathrm{g})$, calycosin $7-\mathrm{O}-\beta$-glucoside $(0.23 \mathrm{mg} / \mathrm{g})$, ammonium glycyrrhizinate $(0.26 \mathrm{mg} / \mathrm{g})$, naringin $(1.80 \mathrm{mg} / \mathrm{g}), \quad 3,6^{\prime}$-disinapoylsucrose $(0.11 \mathrm{mg} / \mathrm{g})$, hesperidin $(0.12 \mathrm{mg} / \mathrm{g})$, neohesperidin $(0.65 \mathrm{mg} / \mathrm{g})$, and astragaloside IV $(0.05 \mathrm{mg} / \mathrm{g})$. The extract being used in this study reached the aforementioned requirements, which ensured the repeatability of biological results.

3.2. XEASD Induces Neurite Outgrowth and Neurofilament Expression of PC12 Cells. To study the trophic effect of XEASD on PC12 differentiation, cultured PC12 cells were treated with XEASD extract for 48 hours before examination under light microscope. As indicated in Figure 2(a), it was observed that neurites were produced from the cell bodies of PC12 cells by XEASD treatment. To quantify the morphological change, that is, differentiated cells and neurite length, the results showed that after the treatment with XEASD extract at various concentrations on PC12 cells for 48 hours, the differentiated cells at highest response were calculated to $50 \%$ of the total cells in cultures (Figure 2(b), upper panel). As for neurite length, application of XEASD extract onto PC12 cells also increased the number of cells possessing neurite length at $15-30 \mu \mathrm{m}$ and $>30 \mu \mathrm{m}$ (Figure 2(b), lower panel). Apart from the morphological observation, the expression 
<smiles>O=C1CC(c2ccc(O[C@@H]3O[C@H](CO)[C@@H](O)[C@H](O)[C@H]3O)cc2)OC2=CC(O)CCC12</smiles>

1: Liquiritin<smiles>C[C@H]1O[C@H](O[C@H]2[C@@H](Oc3cc(O)c4c(c3)O[C@@H](c3ccc(O)cc3)CC4=O)O[C@H](OO)[C@H](O)[C@H]2O)[C@H](O)[C@@H](O)[C@@H]1O</smiles>

4: Naringin<smiles>COc1ccc([C@@H]2CC(=O)c3c(O)cc(O[C@@H]4O[C@H](CO)[C@@H](O)[C@H](O)[C@H]4C[C@H]4O[C@@H](C)[C@@H](O)[C@H](O)[C@H]4O)cc3O2)cc1O</smiles>

7: Neohesperidin<smiles>COC1CCC(C2COC3C=C(OC4O[C@H](O)[C@@H](O)[C@H](O)[C@H]4O)C=CC3C2=O)C=C1O</smiles>

2: Calycosin 7-O- $\beta$-glucoside

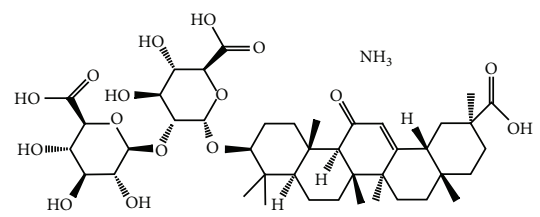

3: Ammonium glycyrrhizinate

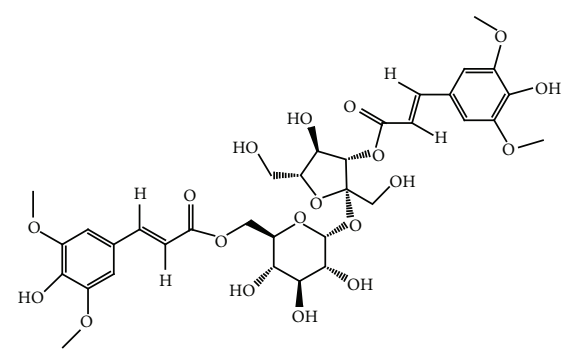

5: 3,6'-disinapoyl sucrose<smiles>COc1ccc([C@H]2CC(=O)c3c(O)cc(O[C@@H]4O[C@H](CC[C@H]5OC(C)[C@H](O)[C@H](O)[C@H]5O)[C@@H](O)[C@H](O)[C@H]4O)cc3O2)cc1O</smiles>

6: Hesperidin

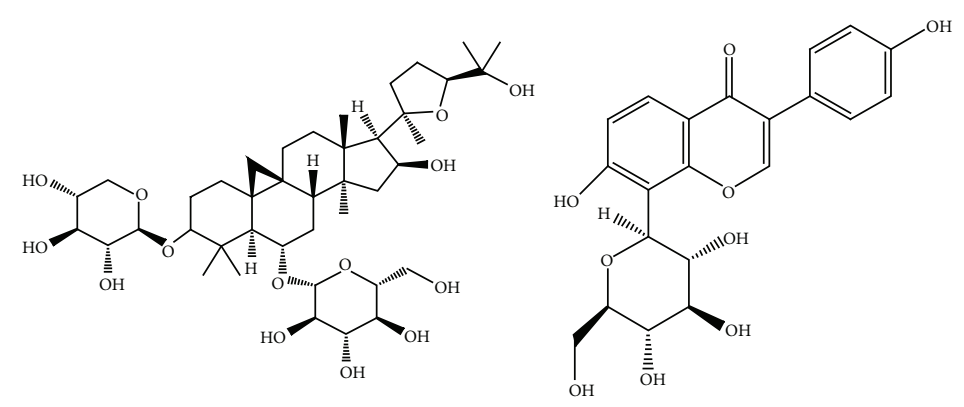

8: Astragaloside IV

ISTD: Puerarin

(a)

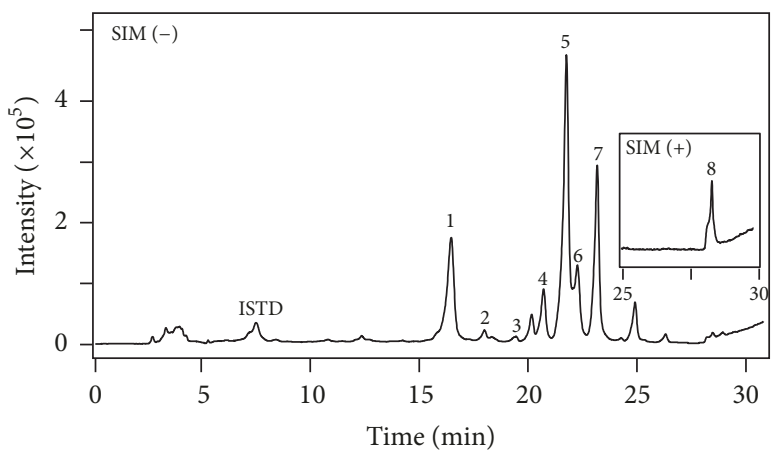

(b)

FIGURE 1: The representative HPLC-MS chromatogram of XEASD. (a) Structures of chemical markers identified in XEASD extract, including liquiritin (1), calycosin 7-O- $\beta$-glucoside (2), ammonium glycyrrhizinate (3), naringin (4), 3,6'-disinapoylsucrose (5), hesperidin (6), neohesperidin (7), astragaloside IV (8), and puerarin (internal standard, ISTD). (b) The representative LC-MS chromatogram of XEASD extract. The LC condition was indicated in the Materials and Methods. The denotations from 1 to 8 in the chromatogram correspond to the chemical markers as shown in (a). The identification of the chemical markers was analyzed by a MS detector in a negative mode except astragaloside IV (8) in a positive mode. Representative chromatograms were shown, $n=3$. 


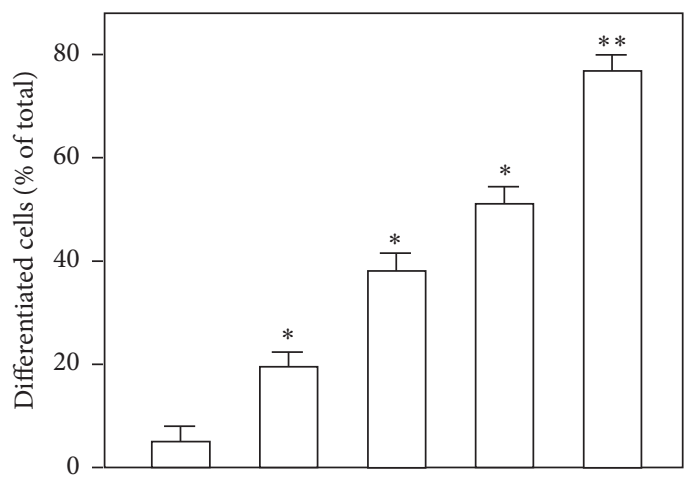

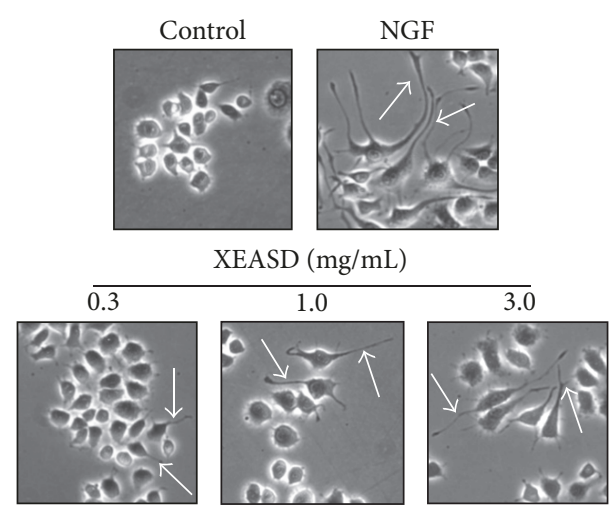

(a)

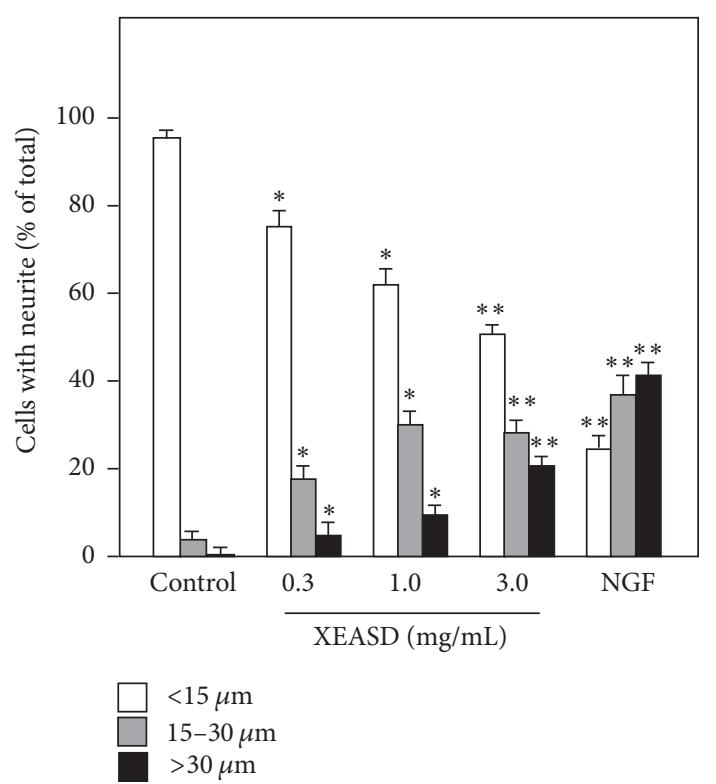

(b)

FIgURE 2: XEASD induces neurite outgrowth of cultured PC12 cells. (a) Cultured PC12 cells were treated with XEASD extract at various concentrations $(0.3-3.0 \mathrm{mg} / \mathrm{mL})$ for 48 hours. NGF $(50 \mathrm{ng} / \mathrm{mL})$ served as the positive control. After treatment, cells were fixed with icecold $4 \%$ paraformaldehyde, and then the neurite outgrowth was measured under microscope. Representative images were shown, $n=5$. Bar $=20 \mu \mathrm{m}$. Arrowheads indicate cell neurite. (b) To quantify the differentiation effect, the $\%$ of differentiated cell numbers (upper panel) and length of neurite (lower panel) were calculated as indicated in the Materials and Methods. The cells were defined as differentiated if one or more neurites were longer than the diameter of cell body and also classified according to their neurite length in $<15 \mu \mathrm{m}, 15-30 \mu \mathrm{m}$, and $>30 \mu \mathrm{m}$. Data are expressed as $\%$ of cells in 100 counted cells. Mean \pm SEM; $n=4$. Statistical comparison was made with the control; ${ }^{*} p<$ $0.05 ;{ }^{* *} p<0.01$.

of neurofilaments including NF68 (at $\sim 68 \mathrm{kDa}$ ), NF160 (at $\sim 160 \mathrm{kDa}$ ), and NF200 (at $\sim 200 \mathrm{kDa}$ ) was determined by a biochemical analysis to evaluate cell differentiation. Application of XEASD extract at various concentrations $(0.3,1.0$, and $3.0 \mathrm{mg} / \mathrm{mL}$ ) stimulated the expressions of NF68, NF160, and NF200, with the highest induction, $\sim 0.5$-fold, 2 -fold, and 3-fold, respectively (Figures 3(a) and 3(b)). NGF served as positive control, which could induce neurite outgrowth and the expression of neurofilament in PC12 cells.

The nuclear transcription factor cAMP responsive element binding protein (CREB) plays a vital role in neuronal differentiation on PC12 cells [9]. The activation of CREB by phosphorylation recruiting the coactivator CREB-binding protein allows its binding onto the promoter and further induces target gene transcription that results in neuronal differentiation. Hence, the inductive role of XEASD in activating the phosphorylation of CREB was studied. As shown in Figure 4(a), XEASD extract stimulated the phosphorylation of CREB at $\sim 43 \mathrm{kDa}$, and its activation could be fully blocked by H89 (Figure 4(b)).

3.3. Antioxidant Activity of XEASD. The DPPH assay is widely used in analyzing the radical scavenging activity known to be involved with oxidation. Thus, the DPPH radical scavenging activity of XEASD was measured. The results indicated that XEASD extract exhibited scavenging free radical activities, and a dose-dependent manner was observed in reactions of DPPH radical with extracts $(0.3-3.0 \mathrm{mg} / \mathrm{mL})$ (Figure 5(a)). Vitamin C (Vit. C), a commercial antioxidant, served as a positive control. Furthermore, to study the 


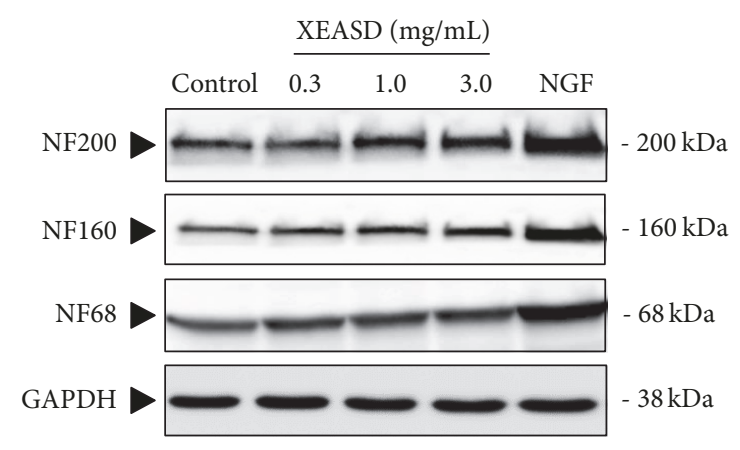

(a)

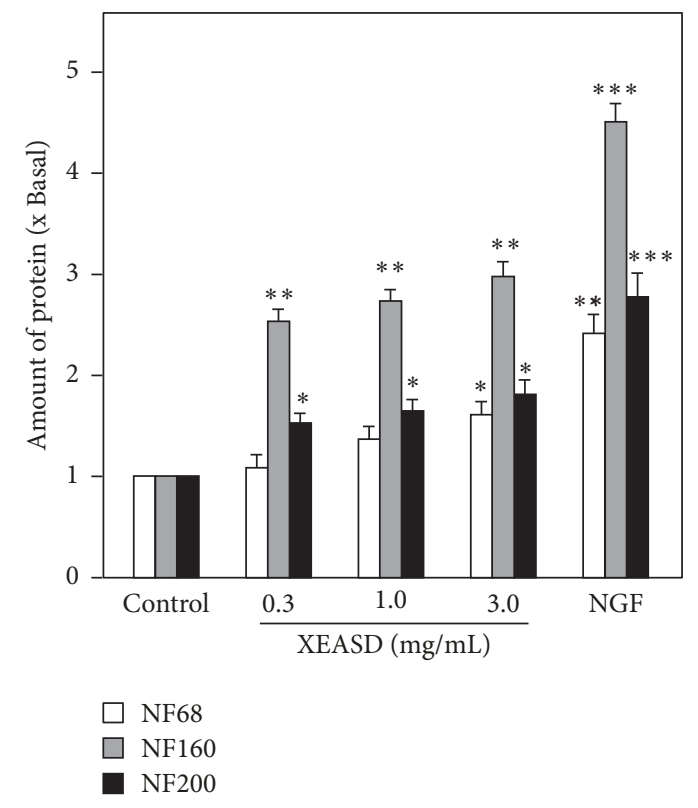

(b)

FIGURE 3: XEASD stimulates neurofilament expression of cultured PC12 cells. (a) XEASD extracts $(0.3-3.0 \mathrm{mg} / \mathrm{mL})$ were applied onto cultured PC12 cells for 48 hours. The cell lysates were collected to determine the expressions of NF68, NF160, and NF200. NGF $(50 \mathrm{ng} / \mathrm{mL})$ served as the positive control. GAPDH served as a loading control. (b) Quantification plot was shown in histograms. Values are expressed as the fold of increase to basal reading (untreated culture, set as 1). Mean \pm SEM; $n=4$. Statistical comparison was made with the control; ${ }^{*} p<0.05 ;{ }^{* *} p<0.01 ;{ }^{* * *} p<0.001$.

transcriptional activity of ARE, PC12 cells were transfected with a luciferase reporter construct (pARE-Luc), containing four AREs derived from the promoter of antioxidant defense genes and having a downstream luciferase reporter gene (Figure 5(b), upper panel). The results demonstrated that application of XEASD extract stimulated the luciferase activity in a dose-dependent manner, with highest response at about 5-fold increase (Figure 5(b), lower panel). tBHQ treatment was used to authenticate the activation of pARELuc, which served as a positive control here (Figure 5).

\section{Discussion}

Neurogenesis is a process known to produce neurons in the brain, by which neuronal differentiation is a vital stage in

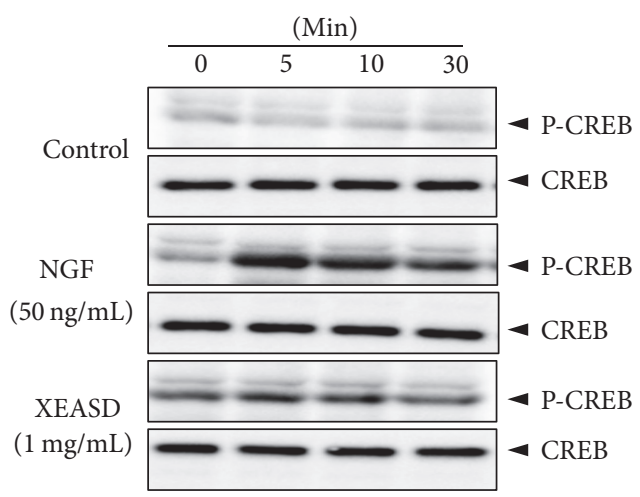

(a)
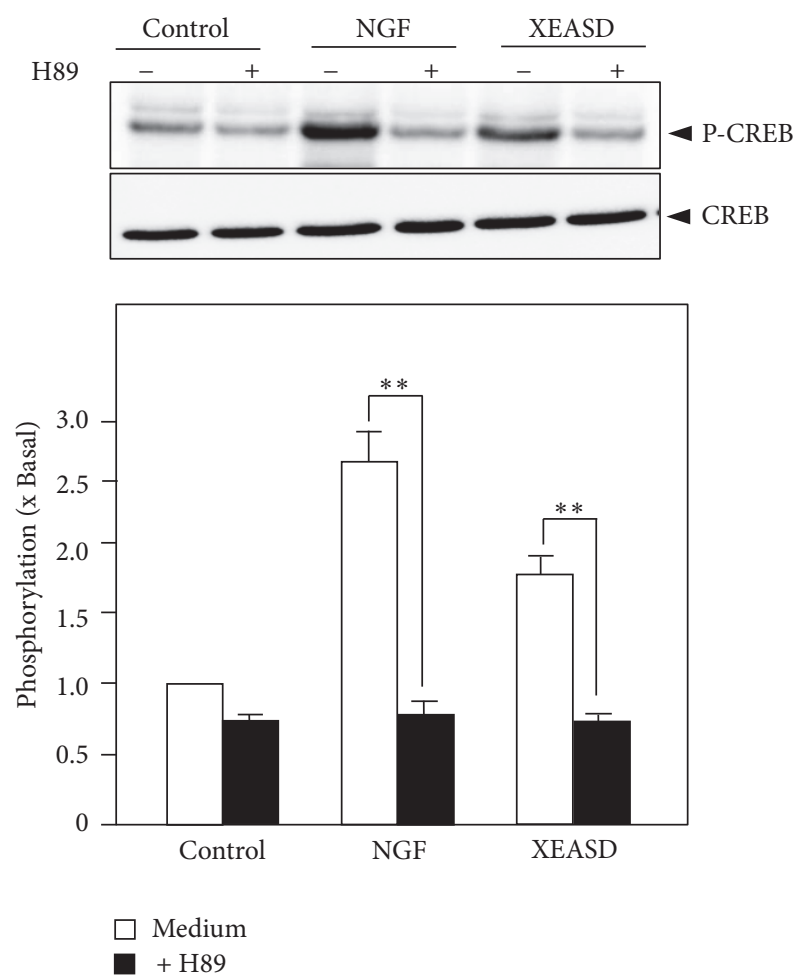

(b)

Figure 4: XEASD induces CREB phosphorylation on PC12 cells. (a) Cultured PC12 cells were serum starved over 5 hours before the treatment with XEASD extract $(1 \mathrm{mg} / \mathrm{mL})$ or NGF $(50 \mathrm{ng} / \mathrm{mL})$ for different time. Total CREB and phosphorylated CREB (both at $43 \mathrm{kDa}$ ) were determined by using specific antibodies. (b) Cultured PC12 cells with serum starvation for over 5 hours were pretreated with or without H89 ( $5 \mu \mathrm{M}$; a PKA inhibitor) for 3 hours prior to the treatment with NGF $(50 \mathrm{ng} / \mathrm{mL})$ and XEASD $(1.0 \mathrm{mg} / \mathrm{mL})$ for 10 minutes. Total CREB and phosphorylated CREB were determined by using specific antibodies (upper panel). Quantification plot was indicated in histograms (lower panel). Values are expressed as the fold of change (x Basal) against the control (no treatment; set as 1). Mean \pm SEM; $n=5$. Statistical comparison was made with the H89treated group; ${ }^{* *} p<0.01$.

developing neurons. During neuron differentiation, the axon and neurite of neuron cell are promoted so as to connect with other neurons to form synapse [10]. Morphological observation including determination of neurite length and 


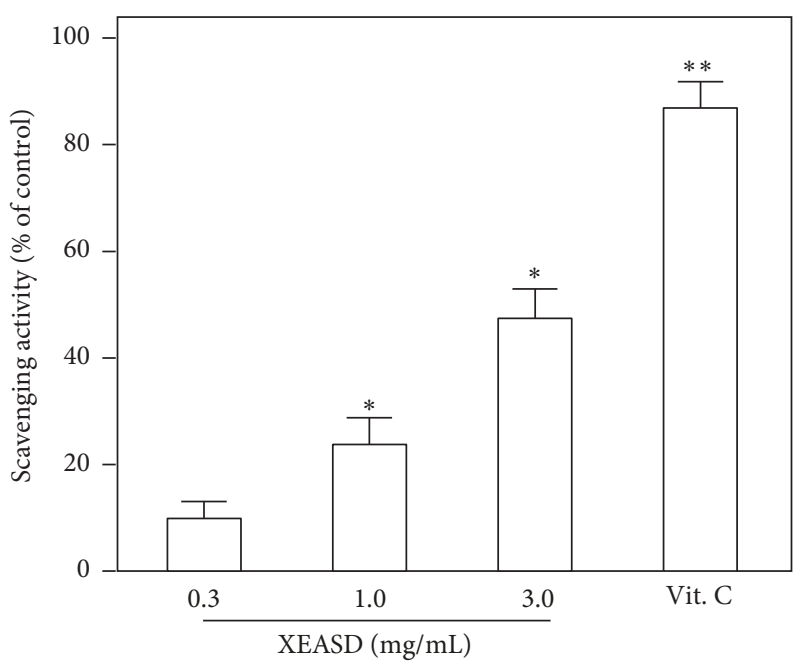

(a)
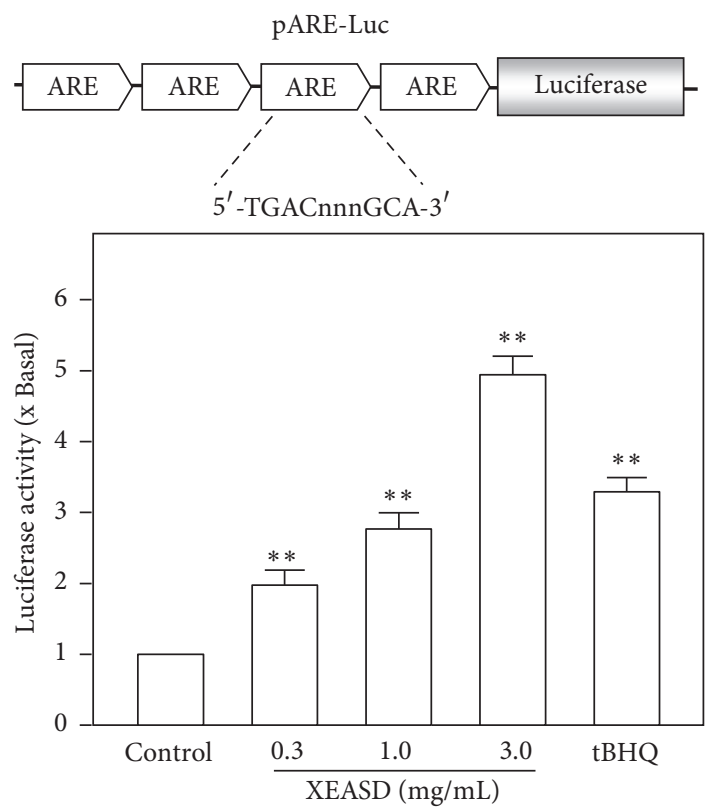

(b)

FIgURE 5: Antioxidation activities of XEASD. (a) An aliquot of $50 \mu \mathrm{L}$ of extracts and vitamin C (Vit. C) was added to a $150 \mu \mathrm{L}$ $\mathrm{DPPH}$ solution. After incubating for $30 \mathrm{~min}$, the decrease in the absorbance of the mixture was determined at $495 \mathrm{~nm}$. The DPPH radical scavenging effect was expressed as \% scavenging relative to control (without samples). (b) A luciferase reporter containing four AREs and a downstream luciferase reporter gene, namely, pARE-Luc, was used as a study tool (upper panel). Cultured PC12 cells, transfected with pARE-Luc, were treated with XEASD extracts $(0.3-3.0 \mathrm{mg} / \mathrm{mL})$ for 24 hours. The cell lysates were subjected to luciferase assay. Values are expressed as the fold of increase to basal reading (untreated culture), and they are in mean $\pm S D$, where $n=4$, each with triplicate samples. Statistical comparison was made with the control; ${ }^{*} p<0.05 ;{ }^{* *} p<0.01$.

calculation of differentiated cell number is widely used to evaluate neuronal differentiation of PC12 cells [11]. Besides, the expressions of neurofilaments including three kinds of molecular weight: $68 \mathrm{KD}, 160 \mathrm{KD}$, and $200 \mathrm{KD}$ were detected
[12]. In our current study, application of XEASD could stimulate the neurite outgrowth of PC12 cells morphologically. In parallel, treatment with XEASD notably induced protein expression of NF160 and NF 200, but to a lesser extent in NF68. Generally, NF68 and NF160 are expressed for the early stage of the differentiation, while NF200 is for the mature stage. Hence, the role of XEASD in neuronal differentiation could be involved in the entire process at both early and late stages. Our further study also demonstrated that XEASD could induce the phosphorylation of CREB. We therefore proposed that PKA-CREB signaling was involved in XEASDinduced neuronal differentiation of PC12 cells.

Currently, induction of neuronal differentiation has been considered as a target for development of antidepressants [10]. Neurotrophin deficiency is reported to involve various neurodegenerative diseases. In depression patient, the neurotrophic factor secretion in the brain was found to reduce, resulting in malfunction of neuron survival, growth, and differentiation [13]. According our findings, XEASD may exert effect of NGF-like or neurotrophic factor stimulating agent in mediating neuronal differentiation of PC12 cells, supporting its application as a potential antidepressant.

On the other hand, oxidative stress is one of the major causes of neuronal cell death in many neurological disorders, that is, depression. Higher animals have developed elaborate defense mechanism, including phase II detoxification enzymes and antioxidant proteins to overcome these insults [14]. Antioxidant response element (ARE) signaling has been demonstrated to play an important role in protecting cells against oxidative stress [15]. We speculated that XEASD possessed free radical scavenging activity. Moreover, XEASD was able to stimulate the transcriptional activity of ARE in cultured PC12 cell. Due to the presence of ARE located on promoters of the defense genes [16], we proposed that the stimulation of the ARE signaling by XEASD may further induce the defense gene expressions of PC12 cells.

XEASD contained numerous herbs, and some of them as well as its major ingredients had been found to possess neurobeneficial effects. Acori Tatarinowii Rhizoma is widely employed clinically within a TCM formula for mental health. Volatile oils were considered as the major active ingredients of Acori Tatarinowii Rhizoma, which had been reported to induce neuronal differentiation [13]. 3,6'-Disinapoyl sucrose found from Polygalae Radix exerted antidepressant-like effect in depressive animal models [17]. Besides, euxanthone isolated from Polygalae Radix was also shown to possess a marked stimulatory action on neurite outgrowth [18]. Triterpenoids from Poria could regulate the expression of 5HT3A receptors in Xenopus oocytes [19]. Flavanoids, such as naringin, hesperidin, and neohesperidin, from Aurantii Fructus and Citri Reticulatae Pericarpium were found to possess neuroprotective effects [20-22]. We hypothesized that the active ingredients in XEASD may exert the neuronal differentiation and antioxidant properties. To support this notation, some of the aforesaid components such as $3,6^{\prime}$ disinapoyl sucrose, naringin, hesperidin, and neohesperidin were identified and quantified within the extract of XEASD by LC-MS analysis, the effects of which need to be further studied. 


\section{Conclusions}

XEASD could induce neurite outgrowth and stimulate the expression of neurofilament in PC12 cells, which supported the enhancement of neurogenesis by XEASD. Besides, XEASD protected cells against oxidative stress in regulating the transcriptional activity of ARE. In conclusion, XEASD could be useful for neurological diseases, in which neurotrophin deficiency and oxidation insult are involved, that is, insomnia and depression. The current studies also provided evidences supporting the clinical usage of XEASD for the treatment of insomnia in children and adolescents.

\section{Disclosure}

Zhonggui Li and Kelly Y. C. Lam are co-first authors.

\section{Conflicts of Interest}

The authors declare that there are no conflicts of interest regarding the publication of this article.

\section{Acknowledgments}

This work was supported by the Shenzhen Science and Technology Plan Project (JCYJ20150401163247216 and ZDSYS201606081515458), Ministry of Human Resources and Social Security of China, Natural Science Foundation of Guangdong Province (2015A030310247), Traditional Chinese Medicine Bureau of Guangdong Province (20151079), and Health and Family Planning Commission of Shenzhen Municipality (201505015).

\section{References}

[1] D. S. Kim, C. L. Lee, and Y. M. Ahn, "Sleep problems in children and adolescents at pediatric clinics," Korean Journal of Pediatrics, vol. 60, no. 5, pp. 158-164, 2017.

[2] L. J. Meltzer, C. Johnson, J. Crosette, M. Ramos, and J. A. Mindell, "Prevalence of diagnosed sleep disorders in pediatric primary care practices," Pediatrics, vol. 125, no. 6, pp. e1410e1418, 2010.

[3] C. Baglioni, G. Battagliese, B. Feige et al., "Insomnia as a predictor of depression: a meta-analytic evaluation of longitudinal epidemiological studies," Journal of Affective Disorders, vol. 135, no. 1-3, pp. 10-19, 2011.

[4] N. Tsuno, A. Besset, and K. Ritchie, "Sleep and depression," Journal of Clinical Psychiatry, vol. 66, no. 10, pp. 1254-1269, 2005.

[5] M. Lopes, A. C. Boronat, Y. Wang, and L. Fu-I, "Sleep complaints as risk factor for suicidal behavior in severely depressed children and adolescents," CNS Neuroscience \& Therapeutics, vol. 22, no. 11, pp. 915-920, 2016.

[6] P. Meerlo, R. E. Mistlberger, B. L. Jacobs, H. C. Heller, and D. McGinty, "New neurons in the adult brain: the role of sleep and consequences of sleep loss," Sleep Medicine Reviews, vol. 13, no. 3, pp. 187-194, 2009.

[7] A. D. Mueller, M. S. Pollock, S. E. Lieblich, J. R. Epp, L. A. M. Galea, and R. E. Mistlberger, "Sleep deprivation can inhibit adult hippocampal neurogenesis independent of adrenal stress hormones," American Journal of Physiology-Regulatory, Integrative and Comparative Physiology, vol. 294, no. 5, pp. R1693-R1703, 2008.

[8] S. L. Xu, R. C. Y. Choi, K. Y. Zhu et al., "Isorhamnetin, a flavonol aglycone from Ginkgo biloba L., induces neuronal differentiation of cultured PC12 cells: potentiating the effect of nerve growth factor," Evidence-Based Complementary and Alternative Medicine, vol. 2012, Article ID 278273, 11 pages, 2012.

[9] D. D. Ginty, A. Bonni, and M. E. Greenberg, "Nerve growth factor activates a Ras-dependent protein kinase that stimulates c-fos transcription via phosphorylation of CREB," Cell, vol. 77, no. 5, pp. 713-725, 1994.

[10] Y. Zhu, X. Duan, X. Cheng et al., "Kai-Xin-San, a standardized traditional Chinese medicine formula, up-regulates the expressions of synaptic proteins on hippocampus of chronic mild stress induced depressive rats and primary cultured rat hippocampal neuron," Journal of Ethnopharmacology, vol. 193, pp. 423-432, 2016.

[11] Z.-A. Chen, J.-L. Wang, R.-T. Liu et al., "Liquiritin potentiate neurite outgrowth induced by nerve growth factor in PC12 cells," Cytotechnology, vol. 60, no. 1-3, pp. 125-132, 2009.

[12] J. Schimmelpfeng, K.-F. Weibezahn, and H. Dertinger, "Quantification of NGF-dependent neuronal differentiation of PC12 cells by means of neurofilament-L mRNA expression and neuronal outgrowth," Journal of Neuroscience Methods, vol. 139, no. 2, pp. 299-306, 2004.

[13] K. Y. C. Lam, J. Chen, C. T. W. Lam et al., "Asarone from Acori tatarinowii Rhizoma potentiates the nerve growth factorinduced neuronal differentiation in cultured $\mathrm{PC} 12$ cells: a signaling mediated by protein kinase A," PLOS ONE, vol. 11, no. 9, Article ID e0163337, 2016.

[14] T. Ishii, K. Itoh, and M. Yamamoto, "Roles of Nrf2 in activation of antioxidant enzyme genes via antioxidant responsive elements," Methods in Enzymology, vol. 348, article no. 18, pp. 182-190, 2002.

[15] J. M. Lee, M. J. Calkins, K. Chan, Y. W. Kan, and J. A. Johnson, "Identification of the NF-E2-related factor-2-dependent genes conferring protection against oxidative stress in primary cortical astrocytes using oligonucleotide microarray analysis," The Journal of Biological Chemistry, vol. 278, no. 14, pp. 12029-12038, 2003.

[16] J.-M. Lee and J. A. Johnson, "An important role of Nrf2ARE pathway in the cellular defense mechanism," Journal of Biochemistry and Molecular Biology, vol. 37, no. 2, pp. 139-143, 2004

[17] Y. Hu, H.-B. Liao, G. Dai-Hong, P. Liu, Y.-Y. Wang, and K. Rahman, "Antidepressant-like effects of 3,6'-disinapoyl sucrose on hippocampal neuronal plasticity and neurotrophic signal pathway in chronically mild stressed rats," Neurochemistry International, vol. 56, no. 3, pp. 461-465, 2010.

[18] M. Naidu, C.-Y. K. Kuan, W.-L. Lo et al., "Analysis of the action of euxanthone, a plant-derived compound that stimulates neurite outgrowth," Neuroscience, vol. 148, no. 4, pp. 915-924, 2007.

[19] J.-H. Lee, Y. J. Lee, J.-K. Shin et al., "Effects of triterpenoids from Poria cocos Wolf on the serotonin type 3A receptormediated ion current in Xenopus oocytes," European Journal of Pharmacology, vol. 615, no. 1-3, pp. 27-32, 2009.

[20] H. D. Kim, K. H. Jeong, U. J. Jung, and S. R. Kim, "Naringin treatment induces neuroprotective effects in a mouse model of Parkinson's disease in vivo, but not enough to restore 
the lesioned dopaminergic system," The Journal of Nutritional Biochemistry, vol. 28, pp. 140-146, 2016.

[21] M. N. Oztanir, O. Ciftci, A. Cetin, and M. A. Aladag, "Hesperidin attenuates oxidative and neuronal damage caused by global cerebral ischemia/reperfusion in a C57BL/J6 mouse model," Neurological Sciences, vol. 35, no. 9, pp. 1393-1399, 2014.

[22] S.-L. Ho, C.-Y. Poon, C. Lin et al., "Inhibition of $\beta$-amyloid aggregation by albiflorin, aloeemodin and neohesperidin and their neuroprotective effect on primary hippocampal cells against $\beta$-amyloid induced toxicity," Current Alzheimer Research, vol. 12, no. 5, pp. 424-433, 2015. 


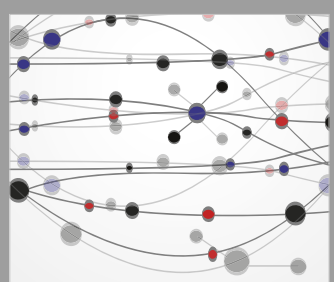

The Scientific World Journal
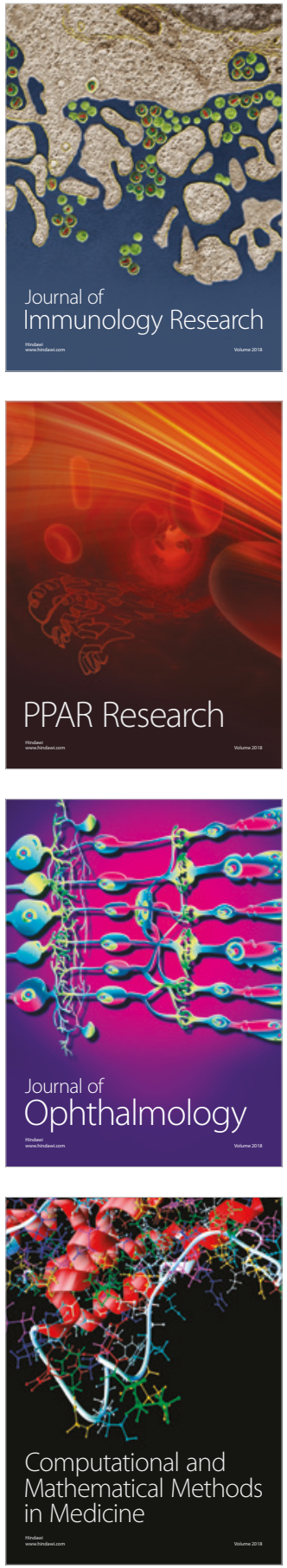

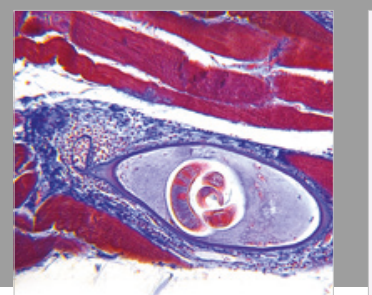

Gastroenterology Research and Practice

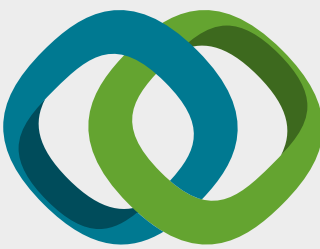

\section{Hindawi}

Submit your manuscripts at

www.hindawi.com
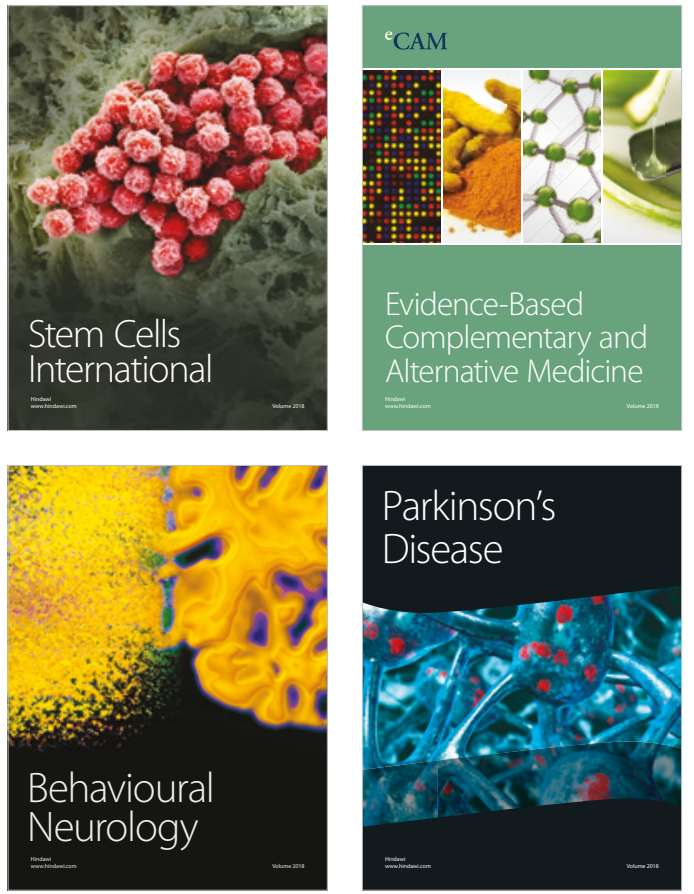

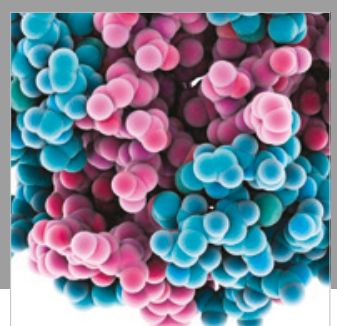

ournal of

Diabetes Research

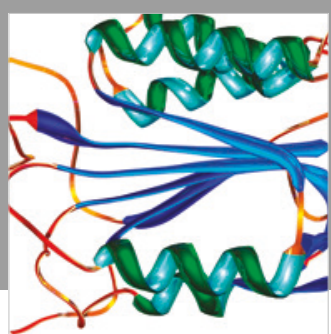

Disease Markers
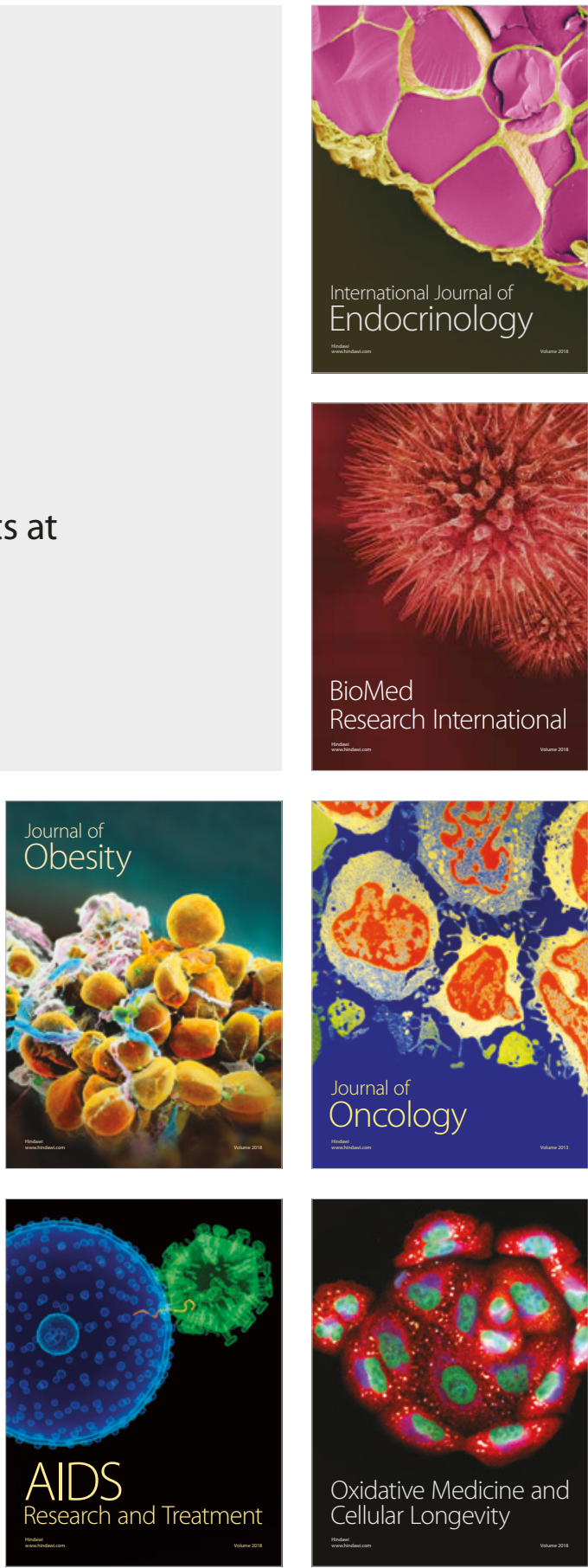